\title{
Prueba cardiopulmonar de ejercicio. Recomendaciones y procedimiento
}

\author{
Silvia Cid-Juárez, José Luis Miguel-Reyes, Arturo Cortés-Télles, \\ Laura Gochicoa-Rangel, Uri de Jesús Mora-Romero, Mónica Silva-Cerón, Luis Torre-Bouscoulet $\bowtie$
}

Instituto Nacional de Enfermedades Respiratorias Ismael Cosío Villegas, Ciudad de México.

Trabajo recibido: 25-IX-2015; aceptado: 08-X-2015

RESUMEN. La prueba cardiopulmonar de ejercicio (PCPE) es una herramienta que permite la evaluación de la respuesta fisiológica tanto en ejercicio submáximo como máximo, brinda información acerca del proceso metabólico, muscular, neurosensorial, cardiovascular y ventilatorio; aunque su uso clínico es cada vez mayor debido a las ventajas clínicas que ofrece en muchos escenarios clínicos y a que los avances en la tecnología han facilitado y agilizado la realización como la interpretación de la prueba, en nuestro medio sigue siendo una prueba poco conocida y no es considerada para el abordaje de los pacientes con intolerancia al ejercicio. El objetivo de este artículo es ofrecer a los neumólogos una visión general de los aspectos prácticos de los principios, de la realización y de la interpretación de la prueba, con la intención de que sea incluida como abordaje de los padecimientos en beneficio de los pacientes.

Palabras clave: Cardiopulmonar, ejercicio, fisiología respiratoria, cardiovascular.

ABSTRACT. Cardiopulmonary exercise testing (CPET) makes it possible to evaluate, simultaneously, the physiological integration of the cardiovascular, respiratory, metabolic, musculoskeletal and neurosensory systems during exercise. CPET provides a physiological response profile that is useful for diagnosis and follow-up of patients in diverse clinical scenarios. Technological advances have facilitated the performance and interpretation of this test; however, in Mexico it is still little-utilized, in part because it is not widely-known. The objectives of this document are, first, to present a general view of the practical aspects of CPET to pneumologists and the medical community as a whole, and, second, to describe the standard procedure for successful test performance. Finally, the text elucidates basic aspects of the interpretation of test results.

Key words: Cardiopulmonary, exercise, respiratory physiology, cardiovascular.

\author{
Abreviaturas: \\ Bloqueo AV: Bloqueo auriculoventricular \\ $\mathrm{CO}_{2}$ : Bióxido de carbono \\ EELV: Volumen pulmonar al final de la espiración \\ EKG: Electrocardiograma \\ EPID: Enfermedad pulmonar intersticial difusa \\ EPOC: Enfermedad pulmonar obstructiva crónica \\ FC: Frecuencia cardíaca \\ $\mathrm{FeCO}_{2}$ : Fracción espirada de bióxido de carbono \\ $\mathrm{FeO}_{2}$ : Fracción espirada de oxígeno \\ $\mathrm{FiO}_{2}$ : Fracción inspirada de oxígeno \\ $\mathrm{H}_{2} \mathrm{O}$ : Agua \\ IC: Capacidad inspiratoria \\ ICC: Insuficiencia cardíaca crónica \\ L: Litros \\ mmHg: Milímetros de mercurio \\ Mseg: Milisegundos \\ $\mathrm{N}_{2}$ : Nitrógeno \\ $\mathrm{O}_{2}$ : Oxígeno \\ $\mathrm{O}_{2} / \mathrm{FC}$ : Pulso de oxígeno \\ ${ }^{\circ} \mathrm{C}$ : Grados centígrados
}

PAD: Presión arterial diastólica

$\mathrm{paO}_{2}$ : Presión arterial de oxígeno

PAS: Presión arterial sistólica

$\mathrm{Pb}$ : Presión barométrica

PECP: Prueba cardiopulmonar de ejercicio

$\mathrm{PETCO}_{2}: \mathrm{CO}_{2}$ al final de la espiración

$\mathrm{PH}_{2} \mathrm{O}$ : Presión de agua

RPM: Revoluciones por minuto

$\mathrm{SaO}_{2}$ : Saturación arterial de oxígeno (medición directa: gasometría arterial)

$\mathrm{SpO}_{2}$ : Saturación arterial de oxígeno (medición indirecta: oximetría de pulso)

T: Tiempo

$\mathrm{Vd} / \mathrm{Vt}$ : Espacio muerto

VE: Ventilación minuto

VE/VVM-1: Reserva ventilatoria

VI: Volumen inspirado

$\mathrm{VO}_{2}$ : Consumo de oxígeno

$\mathrm{VO}_{2}$ máx.: Consumo máximo de oxígeno

$\mathrm{VO}_{2}$ pico: Consumo pico de oxígeno

VVM: Ventilación voluntaria máxima 


\section{INTRODUCCIÓN}

La prueba cardiopulmonar de ejercicio (PCPE) permite evaluar la integración fisiológica al ejercicio de los sistemas cardiovascular, respiratorio, metabólico, musculoesquelético y neurosensorial. Desde el punto de vista clínico, la PCPE nos permite cuantificar la capacidad de ejercicio que tiene un individuo ya sea en condiciones de salud o de enfermedad, explorar los factores limitantes al ejercicio, llevar a cabo el seguimiento funcional de los pacientes mediante la tolerancia al ejercicio, evaluar el pronóstico y la respuesta a las intervenciones realizadas; además, la PCPE permite definir con precisión el riesgo quirúrgico y seleccionar a los candidatos para trasplante de corazón; mediante esta prueba se planifican los programas de rehabilitación cardíaca y pulmonar., ${ }^{1,2} \mathrm{La}$ PCPE ofrece un perfil de respuesta al ejercicio y en raras ocasiones permite establecer un diagnóstico nosológico. Los diagnósticos nosológicos que se pueden establecer en esta prueba incluyen al asma inducido por ejercicio, cardiopatía isquémica e insuficiencia arterial periférica. En el resto de los casos, los resultados de la PCPE deben ser analizados a la luz de los antecedentes del paciente así como de examen físico y de las pruebas fisiológicas obtenidas en condiciones de reposo. Es el conjunto de pruebas diagnósticas lo que más ayuda para integrar el diagnóstico definitivo. Salvo en casos que se desee conocer la capacidad de ejercicio, la PCPE no debe ser considerada una prueba de escrutinio para enfermedad cardíaca o pulmonar. Este manuscrito tiene el propósito de ofrecer al lector aspectos prácticos acerca de la PCPE. Es relevante que el neumólogo moderno conozca las indicaciones de esta prueba y aspectos sencillos de interpretación. Creemos que de esta manera, la PCPE será utilizada de forma más extensa en beneficio de los pacientes y se extenderá el conocimiento acerca de la fisiología dinámica pulmonar en su interacción con la función cardiovascular. Al final del documento presentamos el procedimiento a seguir para llevar a cabo la PCPE. Aunque ya existen recomendaciones internacionales, nosotros aclaramos algunos aspectos poco definidos en guías internacionales lo que podría facilitar la realización de la prueba. A lo largo de este documento observamos la metodología y los procesos de estandarización utilizados a nivel global.

\section{Las indicaciones para realizar una PCPE se resu- men a continuación}

Evaluación de la disnea no explicada por pruebas funcionales estáticas. Aunque las pruebas de función respiratoria y cardíaca en reposo son útiles en el abordaje inicial de la disnea de esfuerzo, no predicen el rendimiento físico ni la capacidad funcional de una persona; además, correlacionan mal con la sintomatología asociada al esfuerzo. Realizar esta prueba permitiría la detección oportuna de algunas enfermedades cardíacas o respiratorias que de otra manera no podrían ser diagnosticadas en reposo. La PCPE es de utilidad para conocer mecanismos fisiopatológicos como isquemia cardíaca, hipertensión arterial reactiva, hiperreactividad bronquial asociada al ejercicio, atrapamiento aéreo, hiperinflación dinámica, anormalidades en el intercambio gaseoso (hipoxemia durante el ejercicio) y acidosis metabólica temprana. ${ }^{3}$ En la esfera psicológica, la PCPE permite evaluar estados de hiperventilación, pánico o ansiedad durante el ejercicio. El desacondicionamiento físico se puede documentar durante la PCPE. Si la PCPE es normal, se garantiza la integridad de la respuesta fisiológica al ejercicio y hace innecesario realizar otros estudios complementarios.

Evaluación funcional en enfermedad cardiovascular. Es recomendable que, siempre y cuando estén compensados, todos los pacientes con insuficiencia cardíaca (IC) de cualquier causa sean sometidos a esta prueba debido a que el consumo máximo de oxígeno ( $\mathrm{VO}_{2}$ max) es el mejor predictor de mortalidad. La PCPE ayuda a seleccionar a los candidatos a trasplante de corazón. ${ }^{4,5}$ La prueba es útil en la prescripción y evaluación de la respuesta a los programas de rehabilitación cardíaca y respiratoria. Al igual que la prueba de esfuerzo «clásica» (en banda sin fin con protocolo de Bruce o Bruce modificado), la PCPE también es útil para demostrar isquemia miocárdica.

Evaluación funcional en enfermedad pulmonar obstructiva crónica (EPOC). Una proporción significativa de pacientes con EPOC mueren de causas diferentes a la insuficiencia respiratoria; más del $20 \%$ fallecen por enfermedades cardiovasculares. Cuando los síntomas asociados al esfuerzo son desproporcionados al daño estructural y funcional estático (GOLD B), la PCPE es ideal para detectar enfermedades coexistentes que estén contribuyendo con la disnea y, en consecuencia, con la limitación funcional. ${ }^{6}$ Algunos de dichos factores incluyen, isquemia miocárdica, alteración vasculopulmonar o disfunción musculoesquelética. La PCPE permite conocer si la hipoxemia contribuye a la disminución de la capacidad funcional al esfuerzo y permite cuantificar de forma precisa la necesidad de $\mathrm{O}_{2}$ suplementario, también permite evaluar la respuesta de la enfermedad a los tratamientos instituidos. ${ }^{6-8}$ 


\section{Evaluación funcional en ejercicio en otras enfer- medades respiratorias}

Asma: La evaluación funcional durante el ejercicio en pacientes con asma puede ser útil para alentar una mayor actividad física y prescribir un programa de ejercicio específico. En quienes se sospecha clínicamente de asma, pero que tienen espirometría en reposo normal, el diagnóstico podrá realizarse si se documenta broncoespasmo, atrapamiento aéreo y obstrucción al flujo aéreo posterior al ejercicio; sin embargo, una respuesta negativa no debe excluir el diagnóstico. ${ }^{9}$ En tales casos se debe de realizar una prueba de reto con metacolina.

Enfermedad pulmonar intersticial difusa (EPID): La detección temprana de alteraciones en el intercambio gaseoso durante el ejercicio, aun cuando las pruebas de función respiratoria estática sean normales, debe de hacernos sospechar en este diagnóstico y realizar estudios complementarios como lo es la tomografía computada de tórax de alta resolución. En pacientes con EPID, las anormalidades en el intercambio de gases, muchas veces sólo evidentes durante la PCPE, suelen preceder a las anormalidades de la mecánica de la respiración. En el seguimiento de los pacientes con EPID, la PCPE es de utilidad debido a que permite analizar la respuesta a las intervenciones terapéuticas y proporciona valor pronóstico.

Enfermedad vascular pulmonar: En pacientes con hipertensión arterial pulmonar, el $\mathrm{VO}_{2}$ max correlaciona con la disminución del gasto cardíaco y con la cantidad del lecho vascular pulmonar que está "disponible». Por lo tanto, el $\mathrm{VO}_{2}$ max estima la gravedad de la enfermedad..$^{10} \mathrm{La}$ eficiencia ventilatoria expresada como el cociente $\mathrm{VE} / \mathrm{VCO}_{2}$ es otro indicador de gravedad y pronóstico en pacientes con hipertensión arterial pulmonar. Mientras mayor es el cociente, mayor es la presión pulmonar y la probabilidad de morir. ${ }^{11}$ La hipertensión pulmonar no es, como lo era hace algunos años, una contraindicación para realizar la PCPE.

Cirugía pulmonar (cáncer pulmonar, reducción de volumen pulmonar, trasplante pulmonar): $\mathrm{EI}$ $\mathrm{VO}_{2}$ max es el principal predictor de complicaciones postoperatorias. ${ }^{12-14}$ El objetivo de realizar la PCPE en pacientes quirúrgicos es someter a estrés al sistema cardiopulmonar para estimar la reserva fisiológica disponible tras la resección quirúrgica; por lo tanto, esta prueba provee de información que no se puede obtener de los estudios en reposo (espirometría y DLCO).

Foramen oval permeable: Este defecto congénito, que cursa con cortocircuito de derecha a izquierda, puede ser diagnosticado cuando el paciente cursa con hipoxemia durante el ejercicio y no mejora tras la administración de oxígeno al 100\%.4,5

Las contraindicaciones para la realización de la PCPE se pueden clasificar en absolutas y relativas, ${ }^{3,15,16}$ éstas se muestran en la tabla 1. La tabla 2 muestra las causas de interrupción de la prueba.

\section{Aspectos técnicos acerca de la prueba:}

\section{Modalidades de la prueba}

Existen dos modalidades de la PCPE comúnmente empleadas: la cinta de correr (banda sin fin) y la bicicleta ergométrica (cicloergómetro) (figura 1). En la mayoría de los casos, el cicloergómetro es el modo preferido de ejercicio; sin embargo, dependiendo de las razones por las que se solicite la PCPE y la disponibilidad del equipo, la banda sin fin puede llegar a ser una alternativa aceptable. En casos particulares, en que esta prueba esté indicada para la evaluación del ejercicio en situaciones especiales (alteraciones en extremidades inferiores o deportistas), se puede disponer de ergómetros específicos (ergómetro de brazos, PCPE portátil, etc.); sin embargo, estas últimas modalidades tienen menor sensibilidad para detectar limitación cardiopulmonar. ${ }^{3,14-16}$ En la tabla 3 se mencionan las principales diferencias entre ambas modalidades.

\section{PCPE en banda sin fin:}

Consiste en una cinta sin fin que es movida por un motor eléctrico sobre la cual el paciente debe caminar a distintas velocidades e inclinación según el protocolo usado.

Ventajas: El caminar es una actividad más familiar que el ciclismo, es más fisiológico, no requiere de aprendizaje previo y permite movilizar una mayor masa muscular. El consumo pico de oxígeno $\left(\mathrm{VO}_{2}\right.$ pico) suele ser $5-10 \%$ más alto en comparación con el obtenido en un cicloergómetro. Es una modalidad altamente recomendada en pacientes con obesidad y cardiopatía.

Desventajas: Existe un mayor riesgo de artefacto en el registro del ECG y de la tensión arterial. Algunos pacientes pueden sentirse inseguros y aferrarse a las barandillas lo que podría subestimar la capacidad de ejercicio. Es más costosa y requiere mayor espacio. ${ }^{3,14-16}$

\section{PCPE en cicloergómetro:}

Es una bicicleta estática cuya carga de trabajo puede ser regulada. Es recomendado que durante la prueba se mantenga un «pedaleo» constante entre 55-65 revoluciones por minuto (rpm). El cicloergómetro puede tener dos tipos de frenado: 
Tabla 1. Contraindicaciones para realizar la prueba cardiopulmonar de ejercicio.

\begin{tabular}{|c|c|}
\hline Absolutas & Relativas \\
\hline $\begin{array}{l}\text { Infarto agudo del miocardio ( } 3-5 \text { días) } \\
\text { Angina inestable } \\
\text { Arritmia no controlada sintomática o con compromiso hemodi- } \\
\text { námico } \\
\text { Síncope } \\
\text { Endocarditis, pericarditis o miocarditis activas } \\
\text { Estenosis aórtica grave sintomática } \\
\text { Insuficiencia cardíaca no controlada } \\
\text { Tromboembolia pulmonar aguda o infarto pulmonar } \\
\text { Trombosis en miembros inferiores } \\
\text { Sospecha de disección aórtica } \\
\text { Asma no controlada } \\
\text { Edema pulmonar } \\
\text { Insuficiencia respiratoria aguda } \\
\text { SpO }{ }_{2} \text { en reposo <85\% (sólo se realizará si cuenta con } \mathrm{O}_{2} \\
\text { suplementario) } \\
\text { Enfermedades agudas no cardiopulmonares que puedan } \\
\text { afectar el resultado de la prueba o puedan agravarse por el } \\
\text { esfuerzo (procesos infecciosos, insuficiencia renal o hepática, } \\
\text { tirotoxicosis) } \\
\text { Alteraciones mentales que no permitan la colaboración del } \\
\text { paciente } \\
\text { Epilepsia no tratada o mal controlada }\end{array}$ & $\begin{array}{l}\text { Enfermedad coronaria } \\
\text { Enfermedad valvular moderada } \\
\text { Hipertensión arterial grave no controlada (PAS > } 200 \text { mmHg, } \\
\text { PAD > } 120 \mathrm{mmHg} \text { ) } \\
\text { Taquiarritmias o bradiarritmias } \\
\text { Bloqueo A-V de alto grado ( } 3: 1,4: 1 \text {, etc.) o } 3^{\circ} \text { grado } \\
\text { Cardiomiopatía hipertrófica } \\
\text { Hipertensión pulmonar grave } \\
\text { Embarazo avanzado o complicado } \\
\text { Desequilibrio electrolítico } \\
\text { Alteración ortopédica que impida realizar el ejercicio }\end{array}$ \\
\hline
\end{tabular}

Bloqueo A-V: Bloqueo auriculoventricular, PAD: Presión arterial diastólica, PAS: Presión arterial sistólica, $\mathrm{SpO}_{2}$ : Saturación de oxígeno, $\mathrm{O}_{2}$ : Oxígeno.

Tabla 2. Motivos para interrumpir la prueba cardiopulmonar de ejercicio.

Dolor torácico sugestivo de isquemia cardíaca

Cambios en el EKG sugestivos de isquemia

Arritmia compleja

Bloqueo $\mathrm{A}-\mathrm{V}$ de segundo o tercer grado

Disminución > $20 \mathrm{mmHg}$ en la PAS del valor más alto

alcanzado durante la prueba

Hipertensión arterial (PAS > 250 mmHg o PAD > 120

$\mathrm{mmHg}$ )

Inicio súbito de palidez o diaforesis

Confusión mental o incoordinación

Signos de insuficiencia respiratoria

Desaturación de $\mathrm{O}_{2}$ grave $(<80 \%)$ cuando se acompañe de

signos y síntomas de hipoxemia

Signos de falla respiratoria

Fatiga, debilidad o claudicación

Bloqueo A-V: Bloqueo auriculoventricular, EKG: Electrocardiograma, PAS: Presión arterial sistólica, $\mathrm{O}_{2}$ : Oxígeno.

Freno electromecánico: Se aplica una resistencia fija al pedaleo regulando la potencia mediante fricción de bandas ajustables. Es un sistema barato, fácil de manejar y de calibrar y permite un registro nítido del electrocardiograma; sin embargo, es difícil cuantificar la carga porque requiere un ritmo constante de pedaleo (próximo a 55-65 rpm). ${ }^{3,14-16}$

Freno electromagnético: Aplica una resistencia precisa y controlada de forma electromagnética. Permite mantener constante la potencia la cual es independiente de la frecuencia de pedaleo. La medición del trabajo es más fiable porque depende poco de la colaboración del paciente. Es más costoso y difícil de calibrar.

Ventajas del cicloergómetro: Se puede cuantificar fácilmente la carga de trabajo. Genera pocos artefactos en la medición de la presión arterial, oximetría de pulso y el registro del EKG. Ocupa poco espacio y relativamente de menor costo que la banda $\sin$ fin. $^{3}$

Desventajas del cicloergómetro: Requiere coordinación motora en las piernas; las personas no familiarizadas con el uso de la bicicleta, pueden mostrar dificultades para mantener un pedaleo constante. Implica esfuerzo para mover las piernas, especialmente en pacientes obesos. Se obtiene un $\mathrm{VO}_{2}$ pico menor comparado con la banda sin fin.

\section{Medición del flujo de aire (sensores de flujo)}

Los sensores de flujo comprenden una gama de dispositivos que son colocados cerca de la boca y detectan 
A

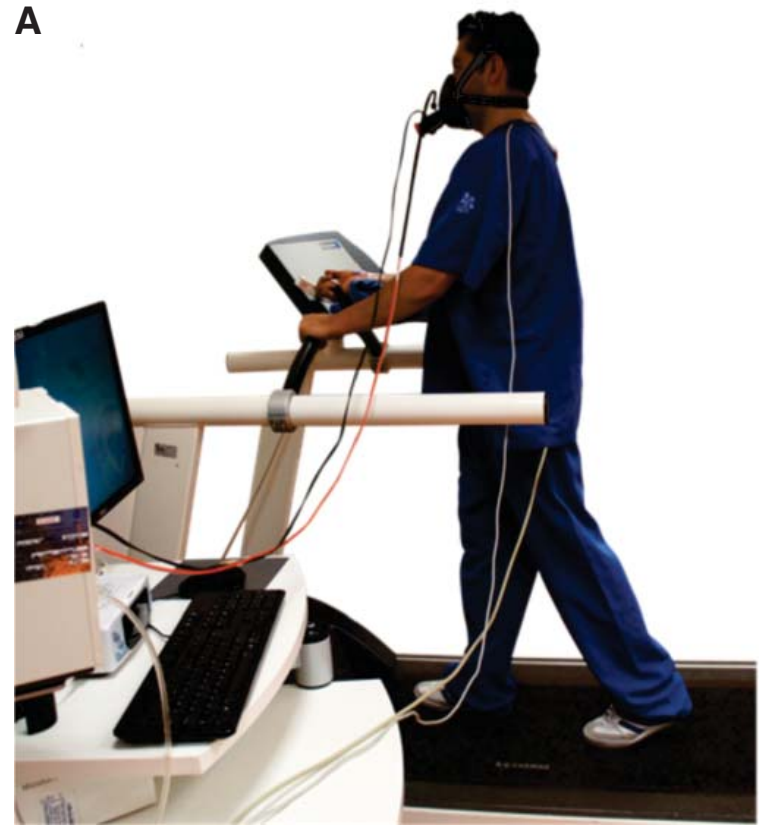

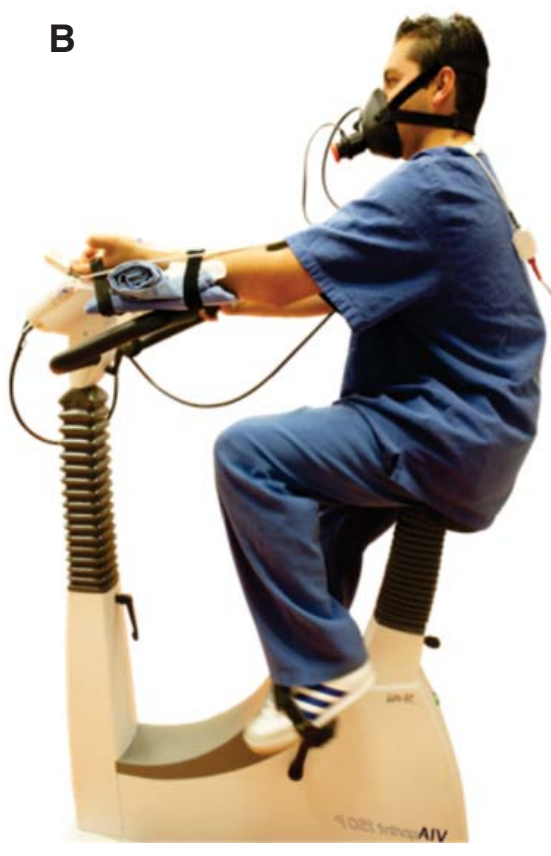

Figura 1.

Modalidades más comunes utilizadas en la clínica para la prueba cardiopulmonar de ejercicio: A. Banda sin fin, B. Cicloergómetro.

Tabla 3. Principales diferencias entre las modalidades de la prueba cardiopulmonar de ejercicio.

Banda sin fin

Caminar/correr es la actividad más cotidiana

Mayor masa muscular es sometida a mayor trabajo

Mayor $\mathrm{VO}_{2}$ pico (> 5-10\%)

Difícil cuantificar con precisión el trabajo, (por lo tanto,

el $\mathrm{VO}_{2}$ pico es un estimado)

Mas artefactos en las mediciones

Más costosa y ocupa más espacio
Cicloergómetro

Requiere coordinación de piernas

Se requiere mayor esfuerzo para mover las piernas

$\mathrm{VO}_{2}$ pico menor

La velocidad a la que se realiza el trabajo es fácilmente cuantifi-

cada

Menos artefactos en las mediciones

Más barata y menor espacio

$\mathrm{VO}_{2}$ : Consumo de oxígeno.

el flujo aéreo de manera bidireccional. En las tablas 4 y 5 se mencionan los diferentes tipos de sensores de flujo y las diferencias entre ellos.

Las características principales de estos dispositivos son: ligeros, con pequeño espacio muerto, de baja resistencia al flujo durante el ejercicio y resistentes a los efectos del vapor de agua o la saliva que pueda llegar a acumularse. ${ }^{3}$

Analizadores de gases: Son instrumentos que miden la composición del gas inspirado y espirado durante la prueba y por lo tanto permiten la evaluación del intercambio de gases. Sus requerimientos son la estabilidad y la rapidez en la respuesta; esta última, a su vez, depende de dos componentes: el retraso en el transporte (tiempo requerido para que el gas llegue desde el lugar de muestreo al analizador) y de la respuesta del análisis (la cinética de la respuesta al cambio en la composición del gas introducido en el analizador).
Ambos retrasos deberían tenerse en cuenta para el cálculo del retraso o rapidez de la señal. Actualmente el software del equipo permite compensar por retraso de transporte de gas (de 0.2 a 0.4 segundos). ${ }^{3}$ Los analizadores de gases son de dos tipos:

- Espectrómetro de masas: Es un instrumento que analiza la composición de los diferentes elementos químicos del aire $\left(\mathrm{O}_{2}, \mathrm{CO}_{2}\right.$, incluso $\left.\mathrm{N}_{2}\right)$. Se prefiere por su exactitud, gran precisión, alta estabilidad y rapidez de respuesta (20-50 mseg). Puede ser configurado para «ignorar» la presión de vapor de agua (proporcionando concentraciones de gas en condiciones de $\mathrm{PH}_{2} \mathrm{O}=0 \mathrm{mmHg}$ ). Tiene un alto costo económico.

- Analizadores independientes de $\mathrm{O}_{2}$ y $\mathrm{CO}_{2}$ : Son comercialmente los más usados. Los más comunes son: para la medición del $\mathrm{CO}_{2}$, los analizadores de 
Tabla 4. Diferentes tipos de sensores de flujo.

\begin{tabular}{|c|c|c|c|}
\hline Neumotacógrafo & Anemómetro de hilo caliente & Tubo pitot & Turbina de volumen \\
\hline $\begin{array}{l}\text { Mide la diferencia de presión } \\
\text { a través de la resistencia } \\
\text { conocida al flujo que ofrece } \\
\text { una malla }\end{array}$ & $\begin{array}{l}\text { Analiza la diferencia de tem- } \\
\text { peratura entre dos filamentos } \\
\text { metálicos colocados en la } \\
\text { dirección del flujo }\end{array}$ & $\begin{array}{l}\text { Mide la diferencia de presión } \\
\text { entre orificios que reciben el } \\
\text { flujo (frontal y perpendicular } \\
\text { respecto a la dirección del } \\
\text { flujo aéreo) }\end{array}$ & $\begin{array}{l}\text { Lector de impulsos luminosos } \\
\text { colocado en la corriente del } \\
\text { flujo, cuenta el número de inte- } \\
\text { rrupciones del destello de luz } \\
\text { Calcula el volumen por un } \\
\text { sistema computarizado }\end{array}$ \\
\hline
\end{tabular}

Tabla 5. Diferencias entre los tipos de sensores de flujo.

\begin{tabular}{|c|c|c|c|c|}
\hline & Neumotacógrafo & $\begin{array}{l}\text { Anemómetro de hilo } \\
\text { caliente }\end{array}$ & Tubo pitot & Turbina de volumen \\
\hline Saliva & Cambia la resistencia & $\begin{array}{l}\text { Cambia la } \\
\text { conductividad }\end{array}$ & No es afectada & $\begin{array}{l}\text { Si se impacta en el lec- } \\
\text { tor cambia la inercia }\end{array}$ \\
\hline Limpieza & Moderada & Moderada & Desechable & Moderada \\
\hline Viscosidad del gas & Cambia la calibración & Independiente & Cambia la calibración & Independiente \\
\hline Densidad del gas & Cambia la calibración & Independiente & Cambia la calibración & Independiente \\
\hline Vapor de agua & $\begin{array}{l}\text { La condensación } \\
\text { cambia la resistencia }\end{array}$ & $\begin{array}{l}\text { Condensación cambia } \\
\text { la conductividad al calor }\end{array}$ & Se afecta poco & $\begin{array}{l}\text { Condensación puede } \\
\text { alterar la masa }\end{array}$ \\
\hline
\end{tabular}

absorción de luz infrarroja; y para la medición del $\mathrm{O}_{2}$ los paramagnéticos y los electroquímicos. Para eliminar el artefacto del vapor de agua, se utiliza un tubo de muestreo fabricado con polímero Nafion (sus grupos sulfidrilo absorben el vapor de agua sin retrasar el análisis de las señales de $\mathrm{O}_{2}$ y $\mathrm{CO}_{2}$ ).

\section{Medición del intercambio respiratorio de gases}

Implica la medición del volumen de $\mathrm{O}_{2}$ y $\mathrm{CO}_{2}$ que entra y sale de los pulmones durante un período dado de tiempo (generalmente intervalos de 1 minuto). El consumo de $\mathrm{O}_{2}$ y la producción de $\mathrm{CO}_{2}$ pueden ser medidos por la diferencia de $\mathrm{O}_{2}$ y $\mathrm{CO}_{2}$ que existe entre el aire inspirado y espirado. ${ }^{3}$ Los métodos usados para esta medición son:

Bolsa meteorológica: Es la técnica más simple para medir la fracción espirada de $\mathrm{O}_{2}$ y $\mathrm{CO}_{2}\left(\mathrm{FEO}_{2}\right.$ y $\left.\mathrm{FECO}_{2}\right)$ a través de la recolección de muestras de gas espirado. Al conocer la $\mathrm{FiO}_{2}$, la medición de $\mathrm{FEO}_{2}, \mathrm{FECO}_{2}$ y del volumen ventilado, permite el cálculo del $\mathrm{VO}_{2}$ y $\mathrm{VCO}_{2}$ :

$$
\begin{gathered}
\mathrm{VO}_{2}=\mathrm{VI}\left(\mathrm{FiO}_{2}\right)-\mathrm{VE}(\mathrm{FEO} 2) / \mathrm{t} \\
\mathrm{VCO}_{2}=\mathrm{FECO}_{2}(\mathrm{VE}) / \mathrm{t}
\end{gathered}
$$

La desventaja que tiene es que el cálculo es inadecuado para aquel protocolo de ejercicio con incrementos rápidos de carga. Este método sólo se usa como una técnica de validación. ${ }^{3}$

Caja de mezclas: Es una caja de $6 \mathrm{~L}$ que permite el muestreo del gas espirado y la medición del VE, con la monitorización continua del $\mathrm{VO}_{2}$ y $\mathrm{VCO}_{2}$. Es adecuado para la medición de gases en un estado estacionario, pero en protocolo de tipo incremental tiene limitaciones para el análisis detallado de los gases respiratorios.

\section{Técnica de respiración a respiración}

Es un sistema automatizado que mide el $\mathrm{VO}_{2}$ sin la necesidad de reunir grandes volúmenes de gas o de realizar cualquier análisis o cálculo a mano. Utiliza un esquema para el procesamiento de las tres principales señales utilizadas en la PCPE: flujo, concentración de $\mathrm{O}_{2}(\%)$ y concentración de $\mathrm{CO}_{2}(\%)$. Con cada espiración, el tiempo espiratorio es dividido en segmentos (cada uno de 10 mseg de duración) y se mide por segmento el VE, $\mathrm{VO}_{2}$ y $\mathrm{VCO}_{2:}$ posteriormente los valores de todos los segmentos se suman para obtener la VE, $\mathrm{VO}_{2}, \mathrm{VCO}_{2}$ de la respiración, al final el resultado es extrapolado a un minuto de respiración. ${ }^{3}$

\section{EKG:}

Durante la prueba se recomienda el registro de:

EKG de 12 derivaciones (en pacientes con sospecha o diagnóstico de enfermedad cardiovascular) para la detección de la enfermedad isquémica (depresiones del segmento ST y cambios en la onda T propios de la isquemia miocárdica) y arritmias secundarias al ejercicio.

EKG de 3 derivaciones (en pacientes jóvenes sin factores de riesgo o antecedentes de enfermedad 
cardíaca) para el registro de la frecuencia cardíaca y establecer el nivel de tolerancia del ejercicio, pero no para explorar la existencia de enfermedad.

Los electrodos deben ser colocados utilizando adhesivos resistentes al sudor y el diseño del circuito electrónico debe estar preparado para evitar los artefactos generados por los movimientos durante el ejercicio. El software debe permitir una monitorización continua de los complejos del EKG en la pantalla durante la PCPE.

Baumanómetro: La toma de la presión arterial suele ser imprecisa durante el transcurso de la prueba de ejercicio debida al incremento del ruido ambiental. Siendo más difícil de medir con la auscultación que con la palpación. Idealmente se debería de colocar un catéter arterial.

Oximetría de pulso: Es útil para la monitorización continua de la $\mathrm{SpO}_{2}$. El error de medición es de $\pm 3 \%$ (pobre perfusión, pigmentación de la piel y movimientos del sensor). No puede medirse la $\mathrm{PaO}_{2}$, por lo que al presentarse una caída de la $\mathrm{PaO}_{2}$ no necesariamente se reflejaría en una desaturación. Se recomienda que en caso de desaturación significativa (caída > 5\%), debería confirmarse con gasometría arterial.

Aunque el oxímetro de pulso tiene un papel central en la monitorización durante el ejercicio, no es útil para estimar los valores de $\mathrm{PaO}_{2}$ arterial. Aunque no forma parte del estándar, en nuestro laboratorio colocamos catéter arterial con fines de realizar un análisis preciso del intercambio de gases.

\section{Otros aditamentos:}

- Escala de Borg: Ésta debe de ser de un tamaño apropiado para que pueda ser de utilidad para pacientes con limitaciones visuales.

- Hoja de recolección de datos.

- Una silla de descanso o cama de exploración para el reposo y vigilancia del paciente posterior a la prueba de esfuerzo cardiopulmonar.

- Desfibrilador externo automático y carro rojo.

- Acceso a teléfono para casos de emergencia.

\section{METODOLOGÍA}

\section{Control de calidad y calibración}

El fabricante debe demostrar la calidad de las mediciones y la información sobre los métodos de medición; sin embargo, el usuario es el responsable de la calibración diaria del equipo. Siempre se debe de disponer de un libro de registro de las calibraciones e incidencias del equipo de medición o cambios en los procedimientos.

\section{Durante el proceso de calibración debe:}

- Registrar diariamente las condiciones ambientales (Presión barométrica, temperatura y vapor de $\mathrm{H}_{2} \mathrm{O}$ ).

- Se debe calibrar:

- El medidor de flujo o volumen con una jeringa de precisión de $3 \mathrm{~L}$.

- Los analizadores de $\mathrm{O}_{2}$ y $\mathrm{CO}_{2}$ en tanques con mezclas de gases de alta precisión, como mínimo en 2 puntos ( 0 y $8 \%$ para $\mathrm{CO}_{2} ; 13$ y $21 \%$ para $\mathrm{O}_{2}$ ).

- El cicloergómetro se debe calibrar (revoluciones por minuto) cada 6 meses o cada vez que se traslade; la banda sin fin también debería ser calibrada, tanto en las revoluciones por minuto como en su grado de inclinación.

- Las mediciones de ventilación y de los gases respiratorios se realizarán mediante la colección de bolsas de gas espirado mezclado (esta calibración puede ser muy laboriosa). La precisión para las variables básicas $\mathrm{VO}_{2}, \mathrm{VCO}_{2}$ y VE debe ser de aproximadamente un $2-3 \%$.

- Para los transductores de presión deberá usarse un manómetro.

\section{METODOLOGÍA}

\section{Aspectos del personal}

El personal que trabajará en los cuidados del equipo y en la realización de las pruebas, debe contar con conocimientos básicos sobre la fisiología del ejercicio. Además, debe estar entrenado de forma adecuada para llevar a cabo la prueba y conocer las técnicas de resucitación cardiopulmonar. Un médico entrenado debe evaluar al paciente antes de la prueba, siempre supervisar la prueba e interpretar los resultados. El grado de supervisión durante la PCPE será determinado por el estado clínico del paciente y las características del protocolo de ejercicio.

\section{METODOLOGÍA}

\section{Selección del protocolo de ejercicio}

El objetivo de la prueba es la de provocar un incremento de los requerimientos energéticos mediante una carga de trabajo controlada para evaluar la reserva funcional de los órganos y sistemas involucrados en la respuesta al ejercicio; a esta forma sistemática de realizar ejercicio se le denomina «protocolo». Los protocolos en la prueba de ejercicio se clasifican de acuerdo con el trabajo realizado en: protocolo incremental (que puede ser 
incremental en rampa o incremental en escalera), protocolo de carga constante y protocolo interválico o discontinuo.

\section{Protocolo incremental}

Es el protocolo más usado, consiste en un incremento progresivo de la carga de trabajo hasta llegar al límite de la tolerancia del paciente que está determinada por la presencia de síntomas; esto permite la evaluación de la respuesta a un amplio espectro de intensidades de ejercicio durante un período relativamente corto de tiempo. Este protocolo está recomendado para evaluar, tanto el perfil de la respuesta biológica durante el ejercicio submáximo como en ejercicio máximo. ${ }^{3,15-18}$ Este protocolo puede ser llevado a cabo en las distintas modalidades de la prueba:

a) En cicloergómetro, el incremento se logra aumentando la resistencia impuesta a través del pedal. En esta modalidad, el protocolo consta de 4 etapas (tabla 6).

b) En banda sin fin, el incremento se logra aumentando la velocidad y la inclinación. En caso de utilizar esta modalidad se aconseja, por su simplicidad, el protocolo modificado de Balke, que consiste en el mantenimiento de una velocidad constante del tapiz (entre 3 y $5.3 \mathrm{~km} / \mathrm{h}$ ) y la programación de incrementos de pendiente (entre el 1 y $2 \%$ por minuto) hasta alcanzar la presencia de síntomas limitantes.

En cardiología, los protocolos incrementales son utilizados para evaluar pacientes con sospecha de cardiopatía isquémica. Los protocolos se clasifican dependiendo de la carga implementada, la inclinación y la duración de la prueba. Algunos son: Protocolo de Bruce, Bruce modificado, Balke o Naughton. La descripción detallada de estos protocolos sale del objetivo de este manuscrito. ${ }^{4,5}$

\section{Protocolos a carga constante}

Cuando un sujeto realiza ejercicio a una carga constante de intensidad moderada, inferior al umbral láctico, suele alcanzar un $\mathrm{VO}_{2}$ estable (estado estacionario) y puede prolongar la duración del ejercicio. Este protocolo consiste en aumentar la carga hasta un nivel a partir del cual se mantiene estable durante un tiempo preestablecido o el tiempo que el paciente tolere. Se mide el tiempo que el paciente «resiste» hacer ejercicio ante una carga constante de trabajo. La utilidad de este tipo de protocolos con carga constante es la de evaluar si el sujeto es capaz de sostener un determinado nivel de ejercicio submáximo durante un período relativamente prolongado de tiempo y la de monitorizar la respuesta a la intervención (rehabilitación cardiopulmonar, broncodilatadores, dispositivos médicos). Mientras más eficaz es la intervención, mayor será el tiempo de «resistencia». Para realizar el protocolo de carga constante, es necesario haber realizado una PCPE con protocolo incremental; así, cuando se realice el protocolo de carga constante, el trabajo máximo al que se someterá al paciente corresponderá al $50-70 \%$ del alcanzado durante el protocolo incremental. ${ }^{3,14-18}$

\section{METODOLOGÍA}

\section{Preparación del paciente}

Antes de empezar a preparar al paciente, deberán tomarse en cuenta algunos aspectos:

\section{Se debe contar con el equipo y material ade- cuado:}

a. Equipo de ejercicio: En nuestro laboratorio es el equipo Oxycon Pro (Jaeger, Germany). El equipo debe tener instalado el sensor de flujo así como los analizadores de $\mathrm{CO}_{2} \mathrm{y} \mathrm{O}_{2}$

b. Equipo de medición del intercambio de gases (bolsa meteorológica, técnica de respiración a respiración o caja de mezclas)

c. Cicloergómetro (calibrado para trabajo en Watts y velocidad)

d. Oxímetro de pulso

e. Baumanómetro y estetoscopio

f. Desfibrilador y carro de emergencia disponibles $y$ funcionales

g. Jeringas de $1 \mathrm{~mL}$

h. Heparina

i. Agujas 25 o $27 \mathrm{G}$

j. Escala de disnea y fatiga Borg

k. Boquillas

I. Mascarilla con arnés

m. Electrodos y software de EKG

n. Gasas

o. Bata de examen médico

p. Escala de Borg de tamaño apropiado para que pueda ser de utilidad para pacientes con limitaciones visuales

q. Hoja de recolección de datos

r. Una silla de descanso o cama de exploración para el reposo y vigilancia del paciente posterior a la prueba

s. Acceso a teléfono para casos de emergencia

t. En aquellos casos en quienes sea necesario 
analizar gases sanguíneos por gasometría arterial se requiere:

i. Gasómetro disponible y calibrado

ii. Personal entrenado y capacitado

iii. Jeringas de insulina

iv. Jeringas de $3 \mathrm{~mL}$

v. Algodón con alcohol

vi. Guantes

vii. Catéter arterial No. 18 (color verde)

viii. Heparina

ix. Lidocaína

x. Soporte de brazos

xi. Campos estériles

xii. Adaptador del catéter para la toma de muestras

xiii. Cinta adhesiva transpore M3

\section{Indicaciones al paciente previo al estudio:}

a. Deberá vestir ropa cómoda y ligera, con zapatos adecuados para realizar ejercicio.

b. Si la prueba se realiza por la mañana o por la tarde, el paciente puede ingerir una comida ligera previamente, pero debe cumplir un ayuno de 2 horas.

c. Debe evitar tabaquismo activo o pasivo y bebidas alcohólicas, al menos 4 horas antes de la prueba.

d. Debe utilizar sus medicamentos de base en la forma habitual.

e. El paciente no deberá haber realizado ejercicio vigoroso un día previo al procedimiento.

\section{Prepare al paciente para la prueba:}

1. El médico y el técnico que realizarán la prueba reciben y se presentan con el paciente. Nota: $\mathrm{La}$ prueba es segura; el riesgo de muerte es de 2-5 por cada 100,000 pruebas realizadas y el riesgo está relacionado con la enfermedad subyacente. Siempre debe estar un médico presente durante la realización de la prueba.

2. El médico debe:

a. Confirmar que el nombre completo coincida con la solicitud médica y con el número de registro.

b. Registrar el (los) diagnóstico (s) y las razones por las cuales solicitan el examen.

c. Realizar un breve interrogatorio y exploración física tratando de identificar posibles contraindicaciones o datos clínicos de exacerbación de enfermedad que no permitan realizar la prueba. d. Registrar el uso de medicamentos (broncodilatadores, antihipertensivos y beta bloqueadores), de estudios paramédicos (función pulmonar, gases arteriales, radiografía del tórax, química sanguínea, etc.) y de síntomas actuales (dolor en tórax, disnea, sibilancias, etc.)

e. Explicar brevemente el procedimiento y responder las inquietudes del paciente, entregándose consentimiento informado (describiendo en forma exacta el procedimiento con sus potenciales riesgos incluyendo la muerte) para que sea firmado por el paciente en presencia de uno o dos testigos. La explicación debe incluir:

i. Información sobre la prueba y del equipo utilizado

ii. Instrucciones de cómo comunicarse con el personal

iii. Los motivos de interrupción de la prueba

iv. Ilustrar cómo efectuar el pedaleo en el cicloergómetro, manteniendo un pedaleo durante toda la prueba y a una velocidad constante (55-65 rpm).

3. Se registra el peso, la estatura y la edad en años cumplidos al día de la prueba.

4. Debe realizarse en reposo una espirometría forzada de acuerdo con los estándares ATS/ERS 2005 y se debe de medir la capacidad inspiratoria y la ventilación voluntaria máxima.

5. Se colocarán los electrodos de 12 derivaciones con la modificación del electrocardiograma estándar de Mason-Likar que consiste en colocar los electrodos de las extremidades al tórax con la finalidad de reducir los artefactos por movimiento. Los electrodos de los brazos se colocarán en el punto más lateral de las fosas infraclaviculares y los electrodos de las piernas deben quedar por arriba de la cresta ilíaca anterior y por debajo de la caja costal. Los electrodos precordiales se colocarán en la forma habitual del electrocardiograma. Se requiere de una colocación meticulosa de los electrodos para disminuir la interferencia.

6. Se colocará catéter arterial en arteria radial de la mano no dominante siempre y cuando la prueba de Allen sea positiva (figura 2).

7. Se le pedirá al paciente que suba al cicloergómetro; verificando que la altura del asiento sea la adecuada y que los pies se encuentren sujetos firmemente a los pedales. Se le pide al paciente que realice una práctica breve con el cicloergómetro antes de iniciar la prueba para que se sienta confortable en el cicloergómetro. 
8. Coloque el brazalete del esfigmomanómetro y oxímetro de pulso y registre las mediciones en reposo.

9. Es importante mencionar que la medición de la presión arterial se puede realizar de forma directa por método invasivo a través del catéter intraarterial conectado a un transductor de presión calibrado para la medición continua.

10. Coloque la boquilla con su correspondiente mascarilla, ajustándola para evitar fugas y solicitará al paciente que respire normalmente (figura 3).

\section{Explique la prueba antes de iniciar con el pro- cedimiento:}

a. El propósito del estudio: es una prueba de esfuerzo máximo limitada por síntomas (fatiga, disnea, dolor torácico, dolor de piernas o no poder mantener el pedaleo constante). Se debe insistir en la necesidad de alcanzar el objetivo del examen: lograr frecuencia cardíaca máxima, trabajo máximo o desarrollo de síntomas limitantes.

b. Todo el tiempo de la prueba se respirará por la boquilla (o mascarilla) en la que han realizado las otras pruebas.

c. Se enseñará la Escala de Borg y registrará los valores de reposo para disnea y fatiga de miembros inferiores; se explicará que estas mediciones serán obtenidas también durante el ejercicio.

d. Se le recordará por último la importancia de mantener el pedaleo constante (55-65 rpm).

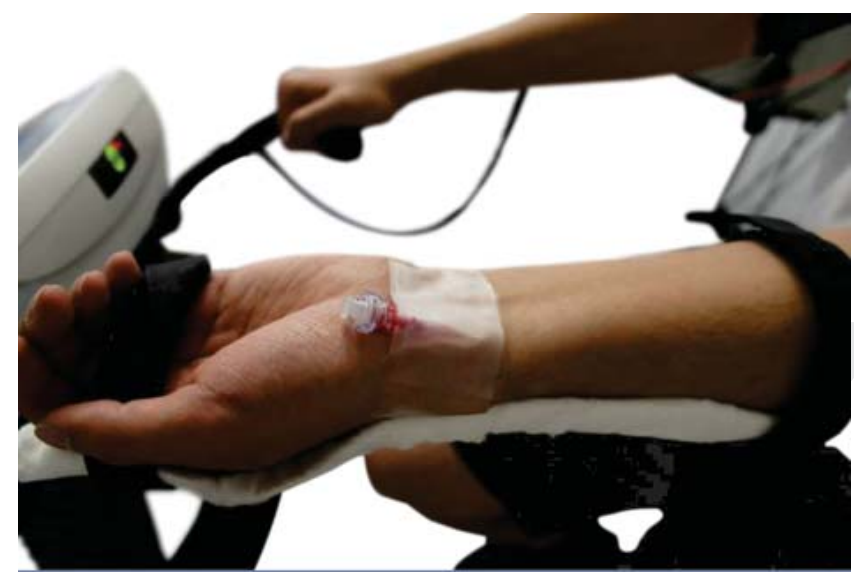

Figura 2. Colocación de la línea arterial, posterior a una prueba de Allen positiva.

\section{Seleccione el protocolo de ejercicio a realizar:}

El protocolo de carga incremental es el utilizado actualmente en el Laboratorio de Fisiología Respiratoria (INER) y consta de las siguientes etapas y mediciones (tabla 6):

\section{Inicie la prueba}

a. Pasada la etapa de reposo (3 minutos) y de pedaleo sin carga (3 minutos) se inicia el ejercicio con carga progresiva.

b. Debe revisarse permanentemente la conexión de los cables, la adecuada posición de la mascarilla y velocidad de pedaleo.

c. Para obtener el esfuerzo máximo del paciente, constantemente se le debe estar motivando para que continúe la prueba.

d. Una vez finalizado el examen se debe permitir que el paciente descanse y se recupere en la silla de descanso o cama de exploración. Comprobando que la tensión arterial, saturación de $\mathrm{O}_{2}$, ritmo y frecuencia cardíaca sean normales o recuperen los valores de reposo antes de permitir su salida. Se recomienda observación por 1 hora.

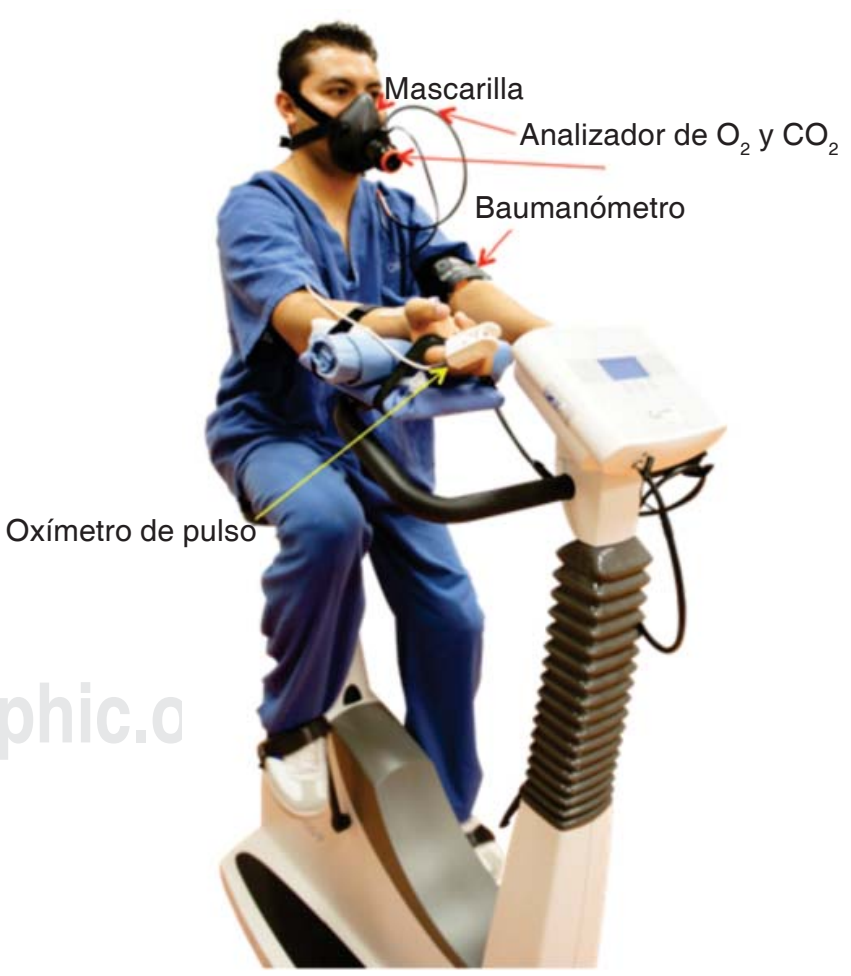

Figura 3. Preparación del paciente previo al inicio de la PCPE. 
Tabla 6. Etapas del protocolo incremental.

\begin{tabular}{|c|c|c|c|}
\hline Etapa & Característica & Duración & Mediciones \\
\hline Reposo & Ausencia total de pedaleo & 3 minutos & $\begin{array}{l}\text { Presión arterial, } \mathrm{SpO}_{2} \text {, } \mathrm{BORG} \text { para dis- } \\
\text { nea y fatiga al final de la etapa } \\
\text { Gasometría arterial si cuenta con línea } \\
\text { arterial }\end{array}$ \\
\hline Calentamiento & $\begin{array}{l}\text { Pedaleo sin carga o mínima } \\
\text { carga }\end{array}$ & 3 minutos & $\begin{array}{l}\text { Presión arterial, } \mathrm{SpO}_{2} \text {, BORG para dis- } \\
\text { nea y fatiga al final de la etapa }\end{array}$ \\
\hline Incremental & $\begin{array}{l}\text { Incremento en rampa de la } \\
\text { resistencia al pedaleo (5-25 ] } \\
\text { Watts/min dependiendo de las } \\
\text { características del paciente) }\end{array}$ & $\begin{array}{l}\text { Variable }(8-10 \mathrm{~min}) \text {, depen- } \\
\text { de del estado funcional del } \\
\text { paciente }\end{array}$ & $\begin{array}{l}\text { Presión arterial, } \mathrm{SpO}_{2} \text {, BORG para } \\
\text { disnea y fatiga cada } 2 \text { minutos y cuando } \\
\text { el paciente alcance umbral anaeróbico y } \\
\text { esfuerzo máximo } \\
\text { Gasometría arterial si cuenta con línea } \\
\text { arterial en el umbral anaeróbico y esfuer- } \\
\text { zo máximo }\end{array}$ \\
\hline Recuperación & $\begin{array}{l}\text { Se retira la resistencia del } \\
\text { cicloergómetro, permitiendo } \\
\text { que el paciente se recupere y } \\
\text { los signos vitales regresen de } \\
\text { forma paulatina a niveles de } \\
\text { reposo }\end{array}$ & Al menos 5 minutos & $\begin{array}{l}\text { Presión arterial, } \mathrm{SpO}_{2}, \mathrm{BORG} \text { para } \\
\text { disnea y fatiga al minuto, a los } 3 \text { y a los } \\
5 \text { minutos }\end{array}$ \\
\hline
\end{tabular}

$\mathrm{SpO}_{2}$ : saturación de oxígeno.

Tabla 7. Parámetros utilizados en el análisis del trabajo, ventilación, función cardiovascular y función muscular para la interpretación de la prueba cardiopulmonar de ejercicio.

\begin{tabular}{|c|c|c|c|}
\hline Trabajo realizado & $\begin{array}{l}\text { Ventilación e intercambio } \\
\text { gaseoso }\end{array}$ & Función cardiovascular & Función muscular \\
\hline Carga de ejercicio (Watts) & Ventilación minuto (VE) & $\begin{array}{l}\text { Frecuencia cardíaca máxima } \\
\text { (FC máxima) }\end{array}$ & Acidosis metabólica \\
\hline Umbral anaeróbico (UA) & Frecuencia respiratoria (FR) & Pulso de $\mathrm{O}_{2}\left(\mathrm{VO}_{2} / \mathrm{FC}\right)$ & Lactato \\
\hline $\begin{array}{l}\text { Tasa de intercambio respirato- } \\
\text { rio (RER) }\end{array}$ & $\begin{array}{l}\text { Reserva respiratoria (VE/ } \\
\text { VVM) }\end{array}$ & Reserva cardíaca & BORG fatiga en piernas \\
\hline $\mathrm{VO}_{2}$ máximo o pico $(\mathrm{L})$ & $\begin{array}{l}\text { Eficiencia de la ventilación } \\
\left(\mathrm{EqCO}_{2} \text { y } \mathrm{EqO}_{2}\right)\end{array}$ & EKG & \\
\hline $\begin{array}{l}\mathrm{VO}_{2} / \mathrm{kg} / \mathrm{min} \\
\mathrm{FC} \text { máxima }\end{array}$ & $\begin{array}{l}\mathrm{SpO}_{2}, \mathrm{PaO}_{2}, \mathrm{PaCO} \mathrm{CO}_{2} \\
\text { Espacio muerto }(\mathrm{Vd} / \mathrm{Vt}) \\
\text { Volúmenes pulmonares diná- } \\
\text { micos (EELV, IC) } \\
\text { BORG para disnea }\end{array}$ & Respuesta presora & \\
\hline
\end{tabular}

EELV: Volumen al final de la espiración, EKG: Electrocardiograma, EqCO : Equivalente de bióxido de carbono, EqO$:$ : Equivalente para oxígeno, FC: Frecuencia cardíaca, FR: Frecuencia respiratoria, IC: Capacidad inspiratoria, RER: Índice de intercambio respiratorio, SpO $:$ Saturación de oxígeno, UA: Umbral anaeróbico, Vd: Volumen muerto, VE: Ventilación minuto, $\mathrm{VO}_{2}$ : Consumo de oxígeno, Vt: Volumen corriente, VVM: Ventilación voluntaria máxima.

\section{Informe:}

En el informe final debe contar con el nombre del paciente y los datos antropométricos, características específicas de la prueba (modalidad y protocolo) y debe contar con la información de las variables en valores absolutos, porcentaje del predicho así como los gráficos de las pruebas respiratorias estáticas así como de la PECP en las diferentes etapas del ejercicio.

Aunque la evaluación de los resultados debe ser siempre sistemática y minuciosa, la interpretación debe estar enfocada al motivo por el cual se indicó el estudio, a los antecedentes patológicos y farmacológicos del paciente. 


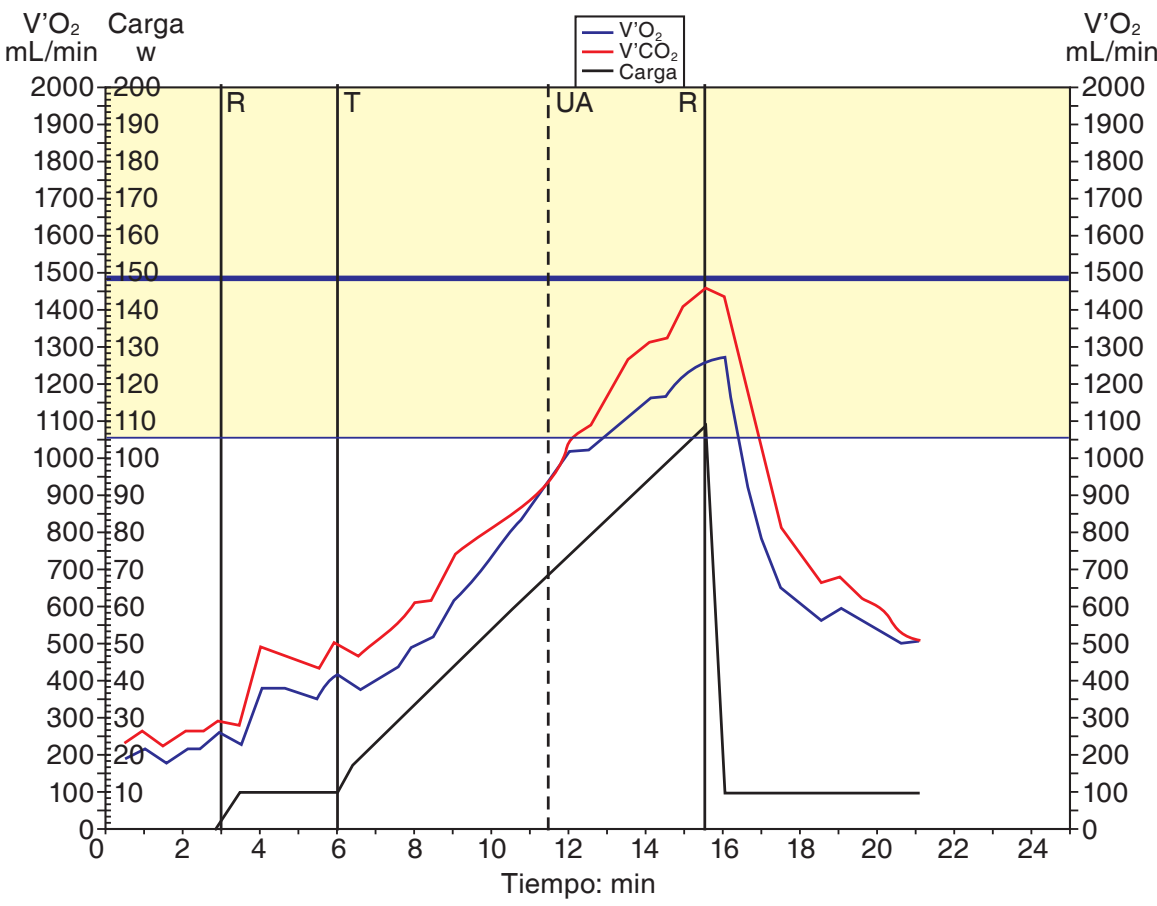

Figura 4.

Curvas de $\mathrm{VO}_{2}$ y $\mathrm{VCO}_{2}$ en relación con el tiempo.

En esta curva se observa el incremento de $\mathrm{VO}_{2}$ (línea azul) y de la $\mathrm{VCO}_{2}$ (línea roja) conforme aumenta la carga impuesta (línea negra).

La línea vertical punteada indica en el momento en que ocurre el umbral anaérobico (ambas pendiente de $\mathrm{VO}_{2} \mathrm{y}$ de $\mathrm{VCO}_{2}$ se cruzan). En sujetos sanos debe estar entre el $40-60 \%$ del $\mathrm{VO}_{2}$ máx. predicho.

Imagen a color en:

www.medigraphic.com/neumologia
A

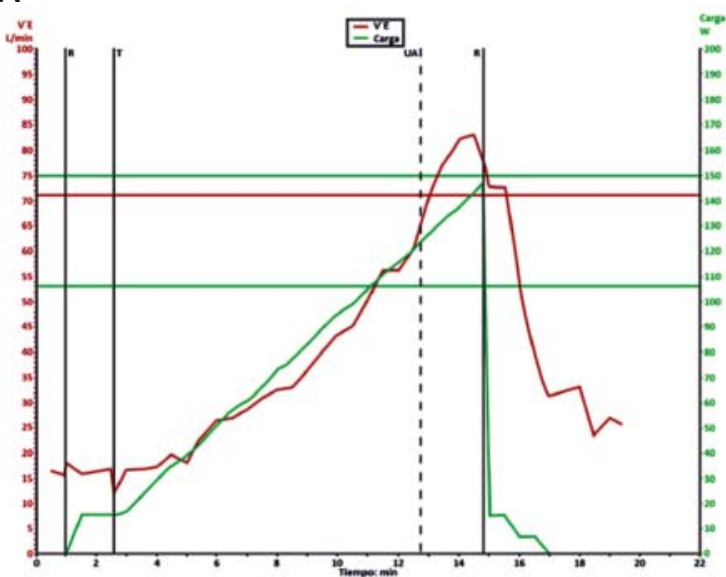

C

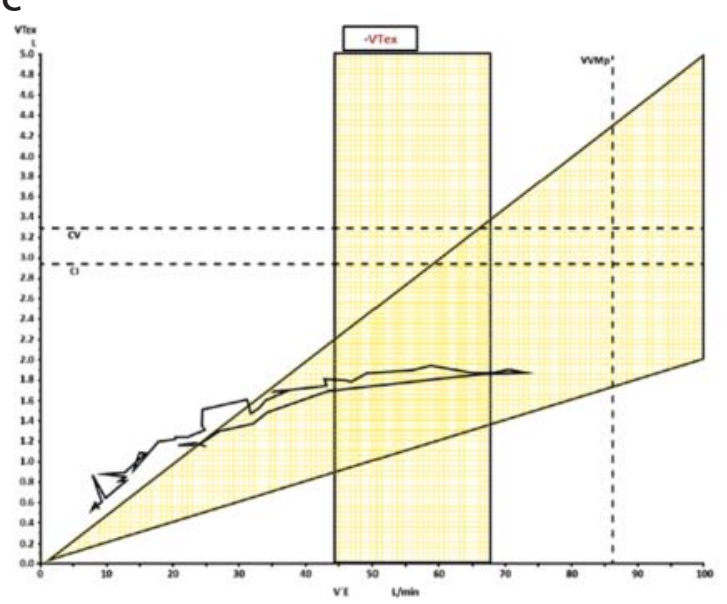

B

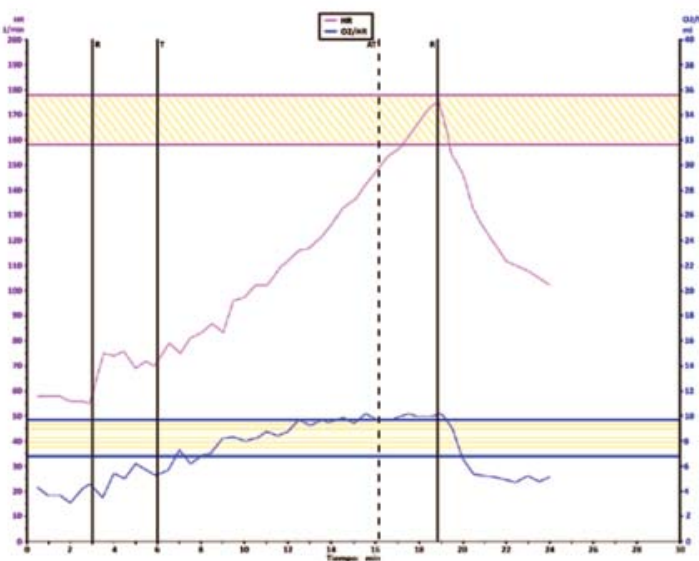

D

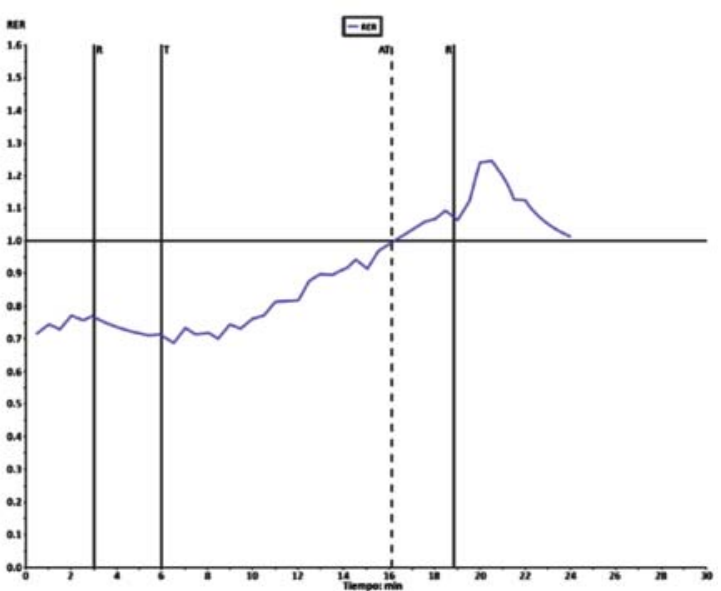

Figura 5.

Gráficas que se reportan en la PCPE:

A. Relación de la carga (Watts) y la VE con el tiempo.

B. Parámetros cardíacos (HR y $\mathrm{VO}_{2} / \mathrm{HR}$ ) en relación con el tiempo.

C. Vt en relación con la VE.

D. UA evaluado por RER (línea vertical punteada).

Imagen a color en:www.medigraphic.com/ neumologia 


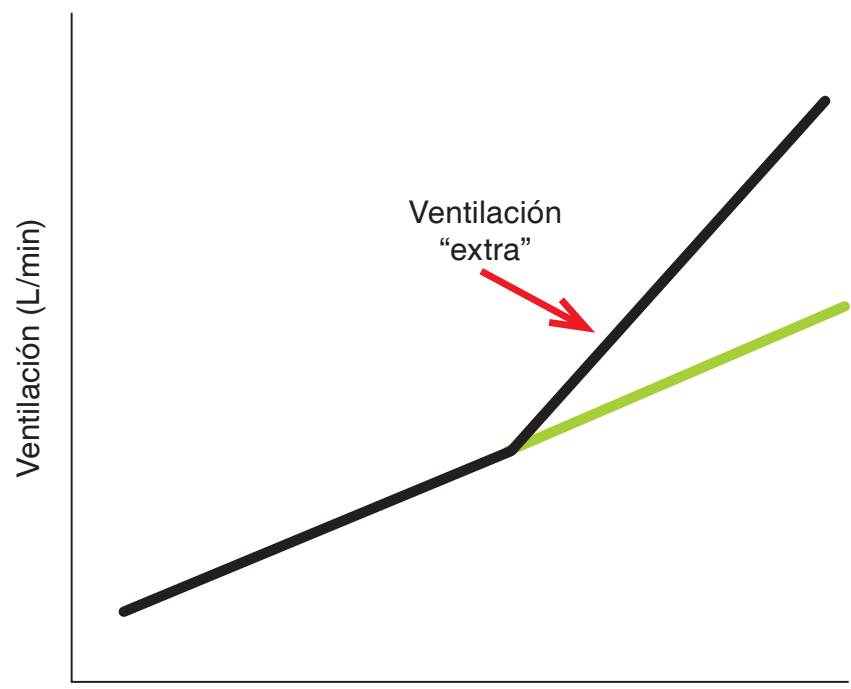

Carga (Watts) o consumo de oxígeno $\left(\mathrm{VO}_{2}\right)$

Figura 6. La ventilación minuto $(\mathrm{Ve})$ en respuesta al trabajo impuesto (Watts) o al consumo de oxígeno logrado $\left(\mathrm{VO}_{2}\right)$.

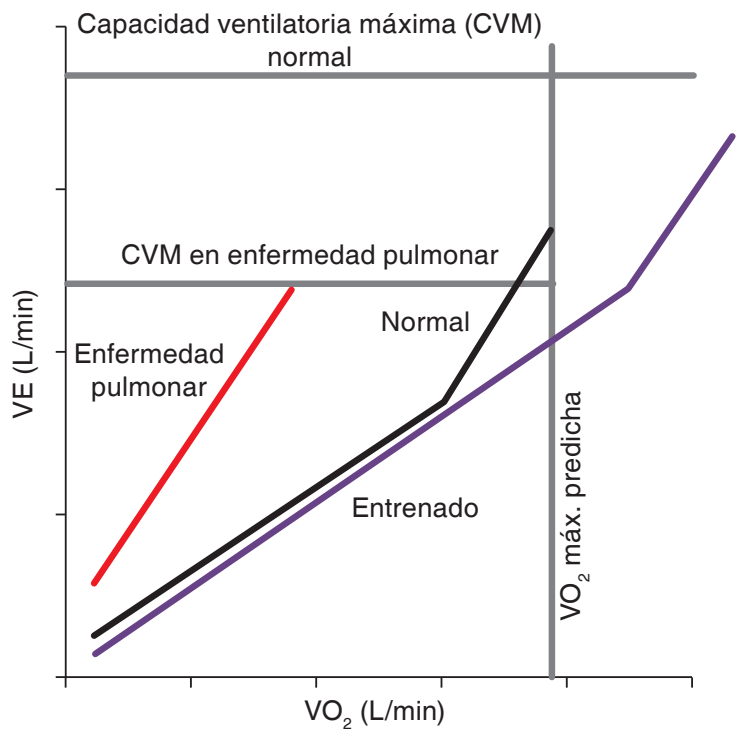

Figura 7. Respuesta respiratoria durante el ejercicio en diferentes situaciones clínicas.

Tabla 8. Patrones básicos de la función cardiopulmonar.

\begin{tabular}{|c|c|c|c|c|c|c|c|c|}
\hline & Sano & $\begin{array}{l}\text { Enfermedad } \\
\text { cardíaca }\end{array}$ & $\begin{array}{c}\text { Enfermedad } \\
\text { obstructiva }\end{array}$ & $\begin{array}{l}\text { Enfermedad } \\
\text { intersticial }\end{array}$ & $\begin{array}{l}\text { Enfermedad } \\
\text { vascular }\end{array}$ & $\begin{array}{l}\text { Neuromus- } \\
\text { cular }\end{array}$ & $\begin{array}{l}\text { Desacondi- } \\
\text { cionamiento }\end{array}$ & Obeso \\
\hline $\mathrm{VO}_{2}$ máx. & $\mathrm{N}$ & $\downarrow$ & $\downarrow$ & $\downarrow$ & $\downarrow$ & $\downarrow$ & $\downarrow$ & $\begin{array}{l}\downarrow \text { (normal } \\
\text { para el peso } \\
\text { ideal) }\end{array}$ \\
\hline $\mathrm{O}_{2} / \mathrm{HR}$ & $\mathrm{N}$ & $\downarrow$ & $\downarrow$ & No $\downarrow$ & $\downarrow$ & $\downarrow$ & $\downarrow$ & $\mathrm{N}$ \\
\hline FC pico & $\mathrm{N}$ & $\begin{array}{l}\text { Variable, nor- } \\
\text { ma en leve }\end{array}$ & $\begin{array}{c}\text { Variable, } \\
\text { norma en } \\
\text { leve }\end{array}$ & $\downarrow$ & $\downarrow$ & No baja & No $\downarrow$ & $\mathrm{N}$ \\
\hline $\begin{array}{l}\text { Reserva } \\
\text { cardíaca }\end{array}$ & $\begin{array}{c}\text { Ausente } \\
(<20 \mathrm{lpm})\end{array}$ & $\begin{array}{c}\text { Ausente o } \\
\text { pequeño } \\
(20 \mathrm{lpm})\end{array}$ & $\begin{array}{c}\text { Grande } \\
(>30 \text { lpm) }\end{array}$ & $\begin{array}{c}\text { Ausente o } \\
\text { pequeño } \\
(20 \mathrm{lpm})\end{array}$ & $\begin{array}{c}\text { Ausente o } \\
\text { pequeño } \\
(20 \mathrm{lpm})\end{array}$ & $\begin{array}{c}\text { Grande } \\
(>30 \mathrm{lpm})\end{array}$ & $\begin{array}{c}\text { Grande } \\
(>30 \mathrm{lpm})\end{array}$ & $\mathrm{N}$ \\
\hline VE máx./VVM & $<0.8$ & $<0.8$ & $>0.8$ & $<0.8$ & $<0.8$ & $<0.8$ & $<0.8$ & $<0.8$ \\
\hline UA & $\begin{array}{l}\text { Presente } \\
\text { (40-60\% de } \\
\mathrm{VO}_{2} \text { máx. } \\
\text { predicho) }\end{array}$ & $\begin{array}{l}\text { Variable } \\
(<30 \% \text { de } \\
\text { VO máx. } \\
\text { predicho })\end{array}$ & $\begin{array}{c}\text { Variable } \\
\text { (40-60\% de } \\
\text { VO máx. } \\
\text { predicho) }\end{array}$ & $\begin{array}{l}\text { Presente } \\
\text { (40-60\% de } \\
\mathrm{VO}_{2} \text { máx. } \\
\text { predicho) }\end{array}$ & $\begin{array}{l}\text { Presente } \\
\text { (40-60\% de } \\
\mathrm{VO}_{2} \text { máx. } \\
\text { predicho) }\end{array}$ & $\begin{array}{l}\text { Presente } \\
\text { (40-60\% de } \\
\text { VO máx. } \\
\text { predicho) }\end{array}$ & $\begin{array}{l}\text { Presente } \\
\text { (40-60\% de } \\
\text { VO máx. } \\
\text { predicho) }\end{array}$ & $\begin{array}{c}\text { Presente } \\
\text { (40-60\% de } \\
\mathrm{VO}_{2} \text { máx. } \\
\text { predicho) }\end{array}$ \\
\hline VD/VT & $\downarrow$ & $\downarrow$ & $\downarrow$ & $\begin{array}{l}\uparrow o \text { sin } \\
\text { cambio }\end{array}$ & $\begin{array}{l}\text { 个o sin } \\
\text { cambio }\end{array}$ & $\downarrow$ & $\downarrow$ & $\downarrow$ \\
\hline $\mathrm{SaO}_{2}$ & $\mathrm{~N}$ & $\mathrm{~N}$ & $\downarrow$ & $\downarrow$ & $\downarrow$ & $\mathrm{N}$ & $\mathrm{N}$ & $\mathrm{N}$ \\
\hline $\mathrm{PaO}_{2}$ & $\mathrm{~N}$ & $N$ & Variable & $\downarrow$ & $\downarrow$ & $N$ & $\mathrm{~N}$ & $\mathrm{~N}$ \\
\hline $\mathrm{PETCO}_{2}$ & $\downarrow$ & $\downarrow$ & $\begin{array}{l}\text { 个osin } \\
\text { cambio }\end{array}$ & $\downarrow$ & $\downarrow$ & $\downarrow$ & $\begin{array}{c}\uparrow \sin \\
\text { cambio }\end{array}$ & $\begin{array}{l}\text { 个osin } \\
\text { cambio }\end{array}$ \\
\hline GA-a & $\mathrm{N}$ & $\mathrm{N}$ & $\uparrow$ & $\uparrow$ & $\uparrow$ & $\mathrm{N}$ & $\mathrm{N}$ & N ot \\
\hline
\end{tabular}

$\mathrm{VO}_{2}$ máx. (consumo máximo de $\mathrm{O}_{2}$ ), $\mathrm{O}_{2} / \mathrm{HR}$ (pulso de $\mathrm{O}_{2}$ ), $\mathrm{FC}$ pico (frecuencia cardíaca pico), VE máx./VVM (reserva ventilatoria), UA (umbral ventilatorio), VD/VT (espacio muerto), $\mathrm{SaO}_{2}$ (Saturación de $\mathrm{O}_{2}$ ), $\mathrm{PaO}_{2}$ (Presión arterial de $\mathrm{O}_{2}$ ), $\mathrm{PETCO}_{2}\left(\mathrm{CO}_{2}\right.$ al final de la espiración), GA-a (gradiente alvéolo-arterial de $\mathrm{O}_{2}$ ). N: normal, $\uparrow$ : aumentado, $\downarrow$ : disminuido, Ipm: latidos por minuto. 
Para la interpretación de la prueba es importante contar con los datos señalados en la tabla 7.

\section{PRINCIPIOS DE INTERPRETACIÓN DE LA PCPE}

Son múltiples los factores responsables de la limitación al ejercicio. Lo importante es lograr identificar cuáles de ellos son los responsables y qué importancia tienen en la respuesta al ejercicio. Tradicionalmente se puede clasificar la limitación como cardiovascular, respiratoria, o incluso de los dos tipos. De la misma forma muchos pacientes se limitarán por síntomas y suspenderán el ejercicio antes de alcanzar alguno de estos límites fisiológicos.

Una visión integrada de las variables que intervienen en la respuesta normal al ejercicio es por lo tanto necesaria y se resaltan algunos puntos que deben tenerse en cuenta en el momento de interpretar la prueba:

1. Determinar la razón por la cual se solicita la prueba.

2. Revisión de la información clínica y los exámenes previos del paciente.

3. Evalúe la calidad de la prueba, el esfuerzo realizado por el paciente y la razón de suspensión de la prueba.

4. Evalúe las variables claves $\mathrm{VO}_{2}$, carga, $\mathrm{FC}, \mathrm{VE}$, $\mathrm{SpO}_{2}$, etc.

5. Evalúe las variables numérica y gráficamente (figuras 4-7).

6. Ponga especial atención a las tendencias o características de las respuestas fisiológicas (respuesta máxima o submáxima).

7. Compare los valores medidos con los de referencia.

8. Determine el tipo de limitación al ejercicio (fisiológica o no fisiológica).

9. Considere qué condiciones clínicas se relacionan con las características de la respuesta al ejercicio.

10. Finalmente correlacione el resultado con el estado funcional del paciente.

\section{Patrones de respuesta al ejercicio}

Aunque la respuesta al ejercicio se modifica por varios factores, se han descrito algunos patrones usuales de comportamiento durante el ejercicio en diversas condiciones clínicas que pueden ser de utilidad en la interpretación de esta prueba ${ }^{1-3,15-18}$ y que se muestran en la tabla 8.

\section{CONCLUSIONES}

La PCPE es la mejor herramienta disponible para evaluar la capacidad de ejercicio tanto en condiciones de salud como de enfermedad. El consumo máximo de oxígeno es un sólido predictor de mortalidad de cualquier causa, especialmente, en pacientes con enfermedades cardiovasculares o respiratorias. La estandarización de la PCPE permite obtener mediciones fisiológicas confiables que permitan analizar el pronóstico y la respuesta a los tratamientos instituidos. Consideramos que el especialista en medicina respiratoria debe contar con las habilidades y destrezas para realizar e interpretar apropiadamente la PCPE.

\section{REFERENCIAS}

1. Wasserman K, Hansen JE, Sue DY, Whipp BJ, Casaburi R. Principles of exercise testing and interpretation: including pathophysiology and clinical applications. 3rd ed. Philadelphia: Lippincott Williams \& Wilkins; 1999.p.xv.

2. Forman DE, Myers J, Lavie CJ, Guazzi M, Celli B, Arena $\mathrm{R}$ Cardiopulmonary exercise testing: relevant but underused. Postgrad Med 2010;122(6):68-86. doi: 10.3810/ pgm.2010.11.2225.

3. American Thoracic Society; American College of Chest Physicians. ATS/ACCP Statement on cardiopulmonary exercise testing. Am J Respir Crit Care Med 2003;167(2):211-277.

4. Gibbons RJ, Balady GJ, Beasley JW, et al. ACC/AHA Guidelines for Exercise Testing. A report of the American College of Cardiology/American Heart Association Task Force on Practice Guidelines (Committee on Exercise Testing). J Am Coll Cardiol 1997;30(1):260-311.

5. Stelken AM, Younis LT, Jennison SH, et al. Prognostic value of cardiopulmonary exercise testing using percent achieved of predicted peak oxygen uptake for patients with ischemic and dilated cardiomyopathy. J Am Coll Cardiol 1996;27(2):345-352.

6. Hamilton AL, Killian KJ, Summers E, Jones NL. Muscle strength, symptom intensity, and exercise capacity in patients with cardiorespiratory disorders. Am J Respir Crit Care Med 1995;152(6 Pt 1):2021-2031.

7. Gallagher CG. Exercise limitation and clinical exercise testing in chronic obstructive pulmonary disease. Clin Chest Med 1994;15(2):305-326.

8. Carlin BW, Clausen JL, Ries AL. The effects of exercise testing on the prescription of oxygen therapy. Chest 1994;106(2):361-365.

9. Garfinkel SK, Kesten S, Chapman KR, Rebuck AS. Physiologic and nonphysiologic determinants of aerobic fitness in mild to moderate asthma. Am Rev Respir Dis 1992;145(4 Pt 1):741-745.

10. Sun XG, Hansen JE, Oudiz RJ, Wasserman K. Exercise pathophysiology in patients with primary pulmonary typertension. Circulation 2001;104(4):429-435.

11. Rubin LJ. Primary pulmonary hypertension. Chest 1993;104(1):236-250.

12. Bolliger $C T$, Jordan $P$, Solèr $M$, et al. Exercise capacity as a predictor of postoperative complications in lung resection candidates. Am J Respir Crit Care Med 1995;151(5):1472-1480.

13. Bolliger CT, Perruchoud AP. Functional evaluation of the lung resection candidate. Eur Respir J 1998;11(1):198-212.

14. Morice RC, Peters EJ, Ryan MB, Putnam JB, Ali MK, Roth JA. Redefining the lowest exercise peak oxygen 
consumption acceptable for lung resection of high risk patients. Chest 1996;110(4 Suppl):161S.

15. Cooper BCh, Storer WT. Exercise testing and interpretation: a practical approach. UK: Cambridge University Press; 2001.p.149-180.

16. Roca RRJ. Clinical exercise testing. Eur Respir Mon 2005;31:146-165.

17. Mezzani A, Agostoni P, Cohen-Solal A, et al. Standards for the use of cardiopulmonary exercise testing for the functional evaluation of cardiac patients: a report from the Exercise Physiology Section of the European Association for Cardiovascular Prevention and Rehabilitation. Eur J Cardiovasc Prev Rehabil 2009;16(3):249-267. doi: 10.1097/HJR.0b013e32832914c8.
18. Luks AM, Glenny RW, Robertson HT. Introduction to cardiopulmonary exercise testing. 2nd Ed. USA: SpringerVerlag; 2013.p.1-10.

\section{$\triangle$ Correspondencia:}

Dr. Luis Torre-Bouscoulet, Subdirector de Investigación Clínica, Instituto Nacional de Enfermedades Respiratorias Ismael Cosío Villegas. Calzada de Tlalpan Núm. 4502,

Colonia Sección XVI, 14080, México, D.F. Teléfono 548717 00, extensión 5360

Correo electrónico: luistorreb@gmail.com

Los autores declaran no tener conflicto de intereses. 